1 Hacettepe Journal of Mathematics and Statistics

h Volume 46 (5) (2017), 829-834

\title{
PS-modules over generalized Malcev-Neumann series
}

\author{
Mohamed A. Farahat ${ }^{* \dagger}$ and Naif S. Al-Harthy ${ }^{\ddagger}$
}

\begin{abstract}
In [1], the first author introduced a new class of extension rings called the generalized Malcev-Neumann series ring $R((S ; \sigma ; \tau))$ with coefficients in a ring $R$ and exponents in a strictly ordered monoid $S$ which extends the usual construction of Malcev-Neumann series rings. The conditions under which the generalized Malcev-Neumann series module $M((S))_{R((S ; \sigma ; \tau))}$ is a PS-module are investigated in the present paper.
\end{abstract}

Keywords: PS-module, generalized Malcev-Neumann series rings.

2000 AMS Classification: Primary 06F05, 16W60, 16D25, 16P60

Received : 03.10.2016 Accepted : 21.12.2016 Doi : 10.15672/ HJMS.2017.433

\section{Introduction}

Throughout this paper $R$ denotes an associative ring with identity and $M_{R}$ a unitary right $R$-module. According to Nicholson and Watters [2], $M_{R}$ is called a $P S$-module if every simple submodule is projective, equivalently if its socle, $\operatorname{Soc}\left(M_{R}\right)$, is projective. Examples of PS-modules include nonsingular modules, regular modules in the sense of Zelmanowitz [3] and modules with zero socle. The class of PS-modules is closed under direct sums and submodules. In [4], Weimin proved that PS-modules are preserved by Morita equivalences and excellent extensions.

For any subset $X$ of $R$, denote

$$
l_{M}(X)=\{m \in M \mid m X=0\} .
$$

*Department of Mathematics and Statistics, Faculty of Science, Taif University, Taif, ElHaweiah, Kingdom of Saudi Arabia (KSA); Mathematics Department, Faculty of Science, AlAzhar University, Cairo, Egypt.

Email : m_farahat79@yahoo.com

$\dagger$ Corresponding Author.

${ }_{\ddagger}^{\ddagger}$ Department of Mathematics and Statistics, Faculty of Science, Taif University, Taif, ElHaweiah, Kingdom of Saudi Arabia (KSA) 
1.1. Theorem ([4]). The following statements are equivalent for a right $R$-module $M_{R}$ : (1) $M_{R}$ is a PS-module.

(2) If $L$ is a maximal right ideal of $R$ then either $l_{M}(L)=0$ or $L=e R$, where $e^{2}=e \in R$.

A left PS-module ${ }_{R} M$ is defined analogously. A ring $R$ is said to be a left PS-ring if ${ }_{R} R$ is a PS-module. Every semiprime ring is a PS-ring. Every PP-ring is a PS-ring (where a ring $R$ is called PP-ring if every principal left ideal is projective). In particular every Baer ring is a PS-ring (where a ring $R$ is called Baer if every left (or right) annihilator is generated by an idempotent). A ring for which every simple singular module is injective is a PS-ring. If $l_{R}(J(R))=0$, then $R$ is a PS-ring. In fact $J(R) \subset L$ for every maximal right ideal so $l_{R}(L)=0$.

The notion of PS-rings is not left-right symmetric (cf. [2]). A ring $R$ is duo if each one-sided ideal of $R$ is a two-sided ideal. As a generalization of left duo rings, a ring $R$ is called weakly left duo if for every $r \in R$ there is a natural number $n(r)$ such that $R r^{n(r)}$ is a two-sided ideal of $R$. A ring $R$ is weakly duo if it is weakly right and left duo. In [4], Weimin proved that a duo ring $R$ is a PS-ring if and only if it is a right PS-ring. In [5], Dingguo generalized this result to weakly duo rings, as follows: A weakly duo reduced ring $R$ is a PS-ring if and only if $R$ is a right PS-ring.

If $R$ is a PS-ring so also are $R[x]$ and $R[[x]]$. The converse of this result is false in general by the following example:

1.2. Example ([2], Example 3.2). If $R=\mathbb{Z}_{4}$, then $R[x]$ and $R[[x]]$ are PS-rings but $R$ is not PS-ring.

The main aim of this paper is to investigate conditions for the generalized MalcevNeumann series module $M((S))_{R((S ; \sigma ; \tau))}$ to be a PS-module.

\section{PS-modules of generalized Malcev-Neumann series rings}

Let $(S, ., \leqslant)$ be a strictly ordered monoid (that is, $(S, \leqslant)$ is an ordered monoid satisfying the condition that, if $s<s^{\prime}$, then $s t<s^{\prime} t$ and $t s<t s^{\prime}$ for $s, s^{\prime}, t \in S$ ). Recall that a subset $X$ of $(S, \leqslant)$ is said to be artinian if every strictly decreasing sequence of elements of $X$ is finite and that $X$ is narrow if every subset of pairwise order-incomparable elements of $X$ is finite. Suppose the two maps $\sigma: S \longrightarrow \operatorname{End}(R)$ and $\tau: S \times S \longrightarrow \mathrm{U}(R)$ (the group of invertible elements of $R)$. Let $A=R((S ; \sigma ; \tau))$ denote the set of all formal sums $f=\sum_{x \in S} a_{x} \bar{x}$ such that $\operatorname{supp}(f)=\left\{x \in S \mid a_{x} \neq 0\right\}$ is an artinian and narrow subset of $S$,

with componentwise addition and the multiplication rule is given by

$$
\left(\sum_{x \in S} a_{x} \bar{x}\right)\left(\sum_{y \in S} b_{y} \bar{y}\right)=\sum_{z \in S}\left(\sum_{\{(x, y) \mid x y=z\}} a_{x} \sigma_{x}\left(b_{y}\right) \tau(x, y)\right) \bar{z},
$$

for each $\sum_{x \in S} a_{x} \bar{x}$ and $\sum_{y \in S} b_{y} \bar{y} \in A$. In order to ensure the associativity, it is necessary to impose two additional conditions on $\sigma$ and $\tau$ namely that for all $x, y, z \in S$,

(i) $\sigma_{x}(\tau(y, z)) \tau(x, y z)=\tau(x, y) \tau(x y, z)$,

(ii) $\sigma_{x} \sigma_{y}=\eta(x, y) \sigma_{x y}$, where $\eta(x, y)$ denotes the automorphism of $R$ defined by

$$
\eta(x, y)(r)=\tau(x, y) r \tau(x, y)^{-1} \text { for all } r \in R .
$$

It is now routine to check that $A=R((S ; \sigma ; \tau))$ is a ring which is called the ring of generalized Malcev-Neumann series. We can assume that the identity element of $A$ is $\overline{1}$, this means that

$$
\sigma_{1}=\operatorname{Id}_{R} \text { and } \tau(x, 1)=\tau(1, x)=1 \text { for any } x \in S .
$$

In this case $r \mapsto r \overline{1}$ is an embedding of $R$ as a subring into $A$. 
For each $f \in A \backslash\{0\}$ we denote by $\pi(f)$ the set of minimal elements of $\operatorname{supp}(f)$. If $(S, \leq)$ is a strictly totally ordered monoid, then $\operatorname{supp}(f)$ is a nonempty well-ordered subset of $S$ and $\pi(f)$ consists of only one element.

Clearly, the above construction generalizes the construction of Malcev-Neumann series rings, in case of $S=G$ (an ordered group), which introduced independently by Malcev and Neumann (see [6] and [7]).

If the order $\leq$ is the trivial order, then $A=R((S ; \sigma ; \tau))$ is the usual crossed product $\operatorname{ring} R[S ; \sigma ; \tau]$. Also, if the monoid $S$ has the trivial order and $\tau$ is trivial, then $A=$ $R((S ; \sigma ; \tau))$ is the usual skew monoid $\operatorname{ring} R[S ; \sigma]$. Whoever if the monoid $S$ has the trivial order and $\sigma$ is trivial, then $A=R((S ; \sigma ; \tau))$ is the usual twisted monoid ring $R[S ; \tau]$. Finally, if the monoid $S$ has the trivial order and $\sigma$ and $\tau$ are trivial, then $A=R((S ; \sigma ; \tau))$ is the usual monoid ring $R[S]$, (see, Sections 3.2 and 3.3 in [8]).

Moreover, if $\alpha$ is a ring endomorphism of $R$ and set $S=\mathbb{Z}_{\geq 0}$ endowed with the trivial order. Define $\sigma: S \longrightarrow$ End $(R)$ via $\sigma(x)=\alpha^{x}$ for every $x \in \overline{\mathbb{Z}}_{\geq 0}$ and $\tau(x, y)=1$ for any $x, y \in \mathbb{Z}$. We have $A=R((S ; \sigma ; \tau))$ is the usual skew polynomial $\operatorname{ring} R[x, \alpha]$. Whoever if $\leq$ is the usual order, then $A=R((S ; \sigma ; \tau))$ is the usual skew power series ring $R[[x, \sigma]]$. If $\alpha$ is a ring automorphism of $R, S=\mathbb{Z}$ and $\leq$ is the usual order, then $A=R((S ; \sigma ; \tau))$ is the usual ring of skew Laurent power series $R\left[\left[x, x^{-1}, \alpha\right]\right]$.

In the same time, if we set also $\sigma(s)=\sigma_{s}=\operatorname{Id}_{R} \in \operatorname{End}(R)$ for all $s \in S$, then it is easy to check that polynomial rings, Laurent polynomial rings, formal power series rings and Laurent power series rings are special cases of $A=R((S ; \sigma ; \tau))$.

If $M_{R}$ is a unitary right $R$-module, then the Malcev-Neumann series module $B=$ $M((S))$ is the set of all formal sums $\sum_{x \in S} m_{x} \bar{x}$ with coefficients in $M$ and artinian and narrow supports, with pointwise addition and scalar multiplication rule is defined by

$$
\left(\sum_{x \in S} m_{x} \bar{x}\right)\left(\sum_{y \in S} a_{y} \bar{y}\right)=\sum_{z \in S}\left(\sum_{\{(x, y) \mid x y=z\}} m_{x} \sigma_{x}\left(a_{y}\right) \tau(x, y)\right) \bar{z},
$$

where $\sum_{x \in S} m_{x} \bar{x} \in B$ and $\sum_{y \in S} a_{y} \bar{y} \in A$. One can easily check that (i) and (ii) ensure that $M((S))$ is a unitary right $A$-module. For each $\varphi \in B \backslash\{0\}$ we denote by $\pi(\varphi)$ the set of minimal elements of $\operatorname{supp}(\varphi)$. If $(S, \leq)$ is a strictly totally ordered monoid, then $\operatorname{supp}(\varphi)$ is a nonempty well-ordered subset of $S$ and $\pi(\varphi)$ consists of only one element.

Let $V$ be a subset of $M_{R}$, then

$$
V((S))=\left\{\varphi=\sum_{x \in S} m_{x} \bar{x} \in B \mid 0 \neq m_{x} \in V \text { and } x \in \operatorname{supp}(\varphi)\right\} .
$$

2.1. Definition ([9]). A right $R$-module $M_{R}$ is called $S$-compatible if, for each $m \in M$, $a \in R$ and $x \in S, m a=0$ if and only if $m \sigma_{x}(a)=0$.

A ring $R$ is called $S$-compatible if $R_{R}$ is an $S$-compatible $R$-module.

Now, we are able to deliver our theorem.

2.2. Theorem. Let $(S, ., \leq)$ be a strictly totally ordered monoid which satisfies the condition that $1 \leq s$ for every $s \in S$ and $M_{R}$ an $S$-compatible module. If $M_{R}$ is a PS-module, then $B_{A}=M((S))_{R((S ; \sigma ; \tau))}$ is a PS-module.

Proof. Let $L$ be a maximal right ideal of $A$. We will show that either $l_{B}(L)=0$ or $L=h A$, where $h^{2}=h \in A$. Since $(S, ., \leq)$ is a strictly totally ordered monoid, $\operatorname{supp}(f)$ is a nonempty well-ordered subset of $S$, for every $0 \neq f=\sum_{x \in S} a_{x} \bar{x} \in A$. We denote by 
$\pi(f)$ the smallest element of support $f$.

For any $s \in S$, set

$$
I_{s}=\left\{a_{s} \in \operatorname{supp}(f) \mid f \in L \text { and } \pi(f)=s\right\} \subset R \text { and } I=\bigcup_{s \in S} I_{s} .
$$

Let $J$ be the right ideal of $R$ generated by $I$. If $J=R$, then there exist $s_{1}, \ldots, s_{n} \in S$, $f_{1}, \ldots, f_{n} \in L$ and $r_{1}, \ldots, r_{n} \in R$ such that

$$
1=a_{s_{1}} r_{1}+\cdots+a_{s_{n}} r_{n},
$$

where $a_{s_{i}} \in I_{s_{i}}$ and $\pi\left(f_{i}\right)=s_{i}$, for every $1 \leq i \leq n$. We will show that $1_{B}(L)=0$. Suppose that $\varphi=\sum_{y \in S} m_{y} \bar{y} \in l_{B}(L)$ and $\varphi \neq 0$. Then $\operatorname{supp}(\varphi)$ is a nonempty well-ordered subset of $S$. Let $t=\pi(\varphi)$. If

$$
m_{t} \sigma_{t}\left(a_{s_{i}}\right) \tau\left(t, s_{i}\right) \neq 0 \text { for some } 1 \leq i \leq n,
$$

then the coefficient of $\varphi f_{i}$ at $t s_{i}=\pi\left(\varphi f_{i}\right)$ is non zero. This means that $\varphi f_{i} \neq 0$ for some $1 \leq i \leq n$, a contradiction. Thus

$$
m_{t} \sigma_{t}\left(a_{s_{i}}\right) \tau\left(t, s_{i}\right)=0 \text { for all } 1 \leq i \leq n .
$$

Since $\tau\left(t, s_{i}\right) \in \mathrm{U}(R)$ and $M_{R}$ is an $S$-compatible module, we get

$$
m_{t} a_{s_{i}}=0 \text { for all } 1 \leq i \leq n .
$$

Consequently,

$$
\begin{aligned}
m_{t} & =m_{t} 1=m_{t}\left(a_{s_{1}} r_{1}+\cdots+a_{s_{n}} r_{n}\right) \\
& =\left(m_{t} a_{s_{1}}\right) r_{1}+\cdots+\left(m_{t} a_{s_{n}}\right) r_{n}=0,
\end{aligned}
$$

a contradiction. Thus $\mathrm{l}_{B}(L)=0$. Suppose that $J \neq R$. We will show that $J$ is a maximal right ideal of $R$. Let $r \in R-J$. If $r \overline{1} \in L$, then $r \in I_{1} \subset I$ and so $r \in J$, a contradiction. Therefore $r \overline{1} \notin L$. Since $L$ is a maximal right ideal of $A$,

$$
A=L+(r \overline{1}) A .
$$

It follows that there exist $f=\sum_{x \in S} a_{x} \bar{x} \in L$ and $g=\sum_{y \in S} b_{y} \bar{y} \in A$ such that $\overline{1}=f+(r \overline{1}) g$. Thus

$$
1=a_{1}+r \sigma_{1}\left(b_{1}\right) \tau(1,1)=a_{1}+r b_{1} .
$$

If $a_{1}=0$, then $1=r b_{1} \in r R$. So $R=J+r R$.

If $a_{1} \neq 0$, then $1 \in \operatorname{supp}(f)$. Since $1 \leq s$ for every $s \in S, \pi(f)=1$. Thus $a_{1} \in I_{1} \subset I \subset J$, which implies that $R=J+r R$.

Hence $J$ is a maximal right ideal of $R$. Since $M_{R}$ is a PS-module, it follows that either $\mathrm{l}_{M}(J)=0$ or $J=e R$, where $e^{2}=e \in R$. According to that we have the following two cases:

Case(1). Suppose that $l_{M}(J)=0$. We will show that $l_{B}(L)=0$. Let $\varphi=\sum_{y \in S} m_{y} \bar{y} \in$ $l_{B}(L)$ and $\varphi \neq 0$. Then $\operatorname{supp}(\varphi)$ is a nonempty well-ordered subset of $S$. Let $s=\pi(\varphi)$. For any $r \in J$, there exist $s_{1}, \ldots, s_{n} \in S, f_{1}, \ldots, f_{n} \in L$ and $r_{1}, \ldots, r_{n} \in R$ such that

$$
r=a_{s_{1}} r_{1}+\cdots+a_{s_{n}} r_{n}
$$

where $a_{s_{i}} \in I_{s_{i}}$ and $\pi\left(f_{i}\right)=s_{i}$, for every $1 \leq i \leq n$. Since $\varphi \in 1_{B}(L), f_{1}, \ldots, f_{n} \in L$, we get $\varphi f_{i}=0$ for every $1 \leq i \leq n$. If

$$
m_{s} \sigma_{s}\left(a_{s_{i}}\right) \tau\left(s, s_{i}\right) \neq 0 \text { for some } 1 \leq i \leq n,
$$


then the coefficient of $\varphi f_{i}$ at $s s_{i}=\pi\left(\varphi f_{i}\right)$ is non zero. This means that $\varphi f_{i} \neq 0$ for some $1 \leq i \leq n$, a contradiction. Thus

$$
m_{s} \sigma_{s}\left(a_{s_{i}}\right) \tau\left(s, s_{i}\right)=0 \text { for all } 1 \leq i \leq n .
$$

Since $\tau\left(s, s_{i}\right) \in \mathrm{U}(R)$ and $M_{R}$ is an $S$-compatible module, we get

$$
m_{s} a_{s_{i}}=0 \text { for all } 1 \leq i \leq n .
$$

Consequently,

$$
m_{s} r=m_{s}\left(a_{s_{1}} r_{1}+\cdots+a_{s_{n}} r_{n}\right)=\left(m_{s} a_{s_{1}}\right) r_{1}+\cdots+\left(m_{s} a_{s_{n}}\right) r_{n}=0 .
$$

Therefore $m_{s} \in l_{M}(J)=0$ and $\pi(\varphi)=s$. Thus $\varphi=0$, a contradiction. Hence $l_{B}(L)=0$.

Case(2). Suppose that $J=e R$, where $e^{2}=e \in R$. We will show that $L=(e \overline{1}) A$, where

$$
(e \overline{1})^{2}=(e \overline{1})(e \overline{1})=e \sigma_{1}(e) \tau(1,1) \overline{1} . \overline{1}=(e \overline{1}) \in A \text {. }
$$

To show that $(e \overline{1}) A \subseteq L$, we need to prove that $(e \overline{1}) \in L$. If $(e \overline{1}) \notin L$, then $A=L+(e \overline{1}) A$. Thus there exist $f \in L$ and $g \in A$ such that $\overline{1}=f+(e \overline{1}) g$. Thus

$$
1=a_{1}+e \sigma_{1}\left(b_{1}\right) \tau(1,1)=a_{1}+e b_{1} .
$$

If $a_{1}=0$, then $1=e b_{1} \in e R=J$, a contradiction.

If $a_{1} \neq 0$, then $1 \in \operatorname{supp}(f)$. Since $1 \leq s$ for every $s \in S, \pi(f)=1$. Thus $a_{1} \in I_{1} \subset I \subset J$, which implies that $a_{1} \in J$ and $J=e R$. Hence $1=a_{1}+e b_{1} \in J+e R=J$, a contradiction. Therefore $(e \overline{1}) \in L$ which implies that $(e \overline{1}) A \subseteq L$.

Conversely, suppose that $f \in L$ and $\pi(f)=s$, then $a_{s} \in I_{s} \subset I \subset J=e R$ and so $a_{s}=e a_{s}$. We claim that $a_{u}=e a_{u}$ for any $u \in \operatorname{supp}(f)$.

Suppose that $a_{v}=e a_{v}$ for each $v<u$. Consider the following element $f_{u} \in A$ defined by:

$$
f_{u}=\sum_{u<v} a_{u} \bar{u}+\sum_{u \geq v} 0 \bar{u}=\sum_{u<v} a_{u} \bar{u}
$$

Thus $\pi\left(f-f_{u}\right)=u$. By hypothesis it is easy to see that $f_{u}=\sum_{u<v} e a_{u} \bar{u}=(e \overline{1}) f_{u} \in$

$(e \overline{1}) A \subset L$. Thus $f-f_{u} \in L$. By analogy with the proof above, it follows that $a_{u}=e a_{u}$, which implies that $f=(e \overline{1}) f \in(e \overline{1}) A$. Thus $L=(e \overline{1}) A$ and the result follows since $(e \overline{1})$ is an idempotent of $A$.

In particular, if we set $M_{R}=R_{R}$ we get the following:

2.3. Corollary. Let $(S, ., \leq)$ be a strictly totally ordered monoid which satisfies the condition that $1 \leq s$ for every $s \in S$ and $R$ an $S$-compatible ring. If $R$ is a right $P S$-ring, then $A=R((S ; \sigma ; \tau))$ is a right $P S$-ring.

\section{References}

[1] M. Farahat, Generalized Malcev-Neumann series modules with the Beachy-Blair condition, Algebra (Hindawi Publishing Corporation), Article ID 595274, (2015), doi:10.1155/2015/595274.

[2] W. Nicholson and J. Watters, Rings with projective socle, Proc. Amer. Math. Soc., 102, (1988), 443-450.

[3] J. Zelmanowitz, Regular modules, Trans. Amer. Math. Soc., 163, (1972), 341-355.

[4] Weimin Xue, Modules with projective socles, Riv. Mat. Univ. Parma, 1(5), (1992), 311-315.

[5] Dingguo Wang, Modules with flat socles and almost excellent extensions, Acta Math. Vietnam., 21(2), (1996), 295-301.

[6] A. Malcev, On embedding of group algebras in a division algebra (in russian), Dokl. Akad. Nauk, (1948), 1499-1501. 
[7] B. Neumann, On ordered division rings. Trans. Amer. Math. Soc., 66, (1949), 202-252.

[8] A. Kelarev, Ring Constructions and Applications, World Scientific, River Edge, New York, 2002.

[9] R. Zhao and Y. Jiao, Principal quasi-baerness of modules of generalized power series, Taiwanese J. Math., 15(2), (2011), 711-722. 